
\title{
25 Research Soure \\ USP13 Genetics and Expression in a Family With Thyroid Cancer
}

\section{Andrea G. Maria ( $\nabla$ andreagutierrez.maria@nih.gov )}

National Institutes of Health https://orcid.org/0000-0002-1615-8254

\section{Bruna Azevedo}

Pontifícia Universidade Católica do Paraná: Pontificia Universidade Catolica do Parana

\section{Nikolaos Settas}

National Institutes of Health

\section{Fady Hannah-Shmouni}

National Institutes of Health

\section{Constantine A. Stratakis}

National Institutes of Health

\section{Fabio R. Faucz}

Pontifícia Universidade Católica do Paraná: Pontificia Universidade Catolica do Parana

\section{Research Article}

Keywords: Papillary thyroid carcinoma, Ubiquitin-specific protease 13, pathogenic variant, oncogene

Posted Date: March 7th, 2022

DOI: https://doi.org/10.21203/rs.3.rs-1402599/v1

License: (c) (1) This work is licensed under a Creative Commons Attribution 4.0 International License. Read Full License 


\section{Abstract}

Purpose: Papillary thyroid carcinoma (PTC) is the most common type of thyroid carcinoma and its incidence has greatly increased in the last 30 years. Ubiquitin-specific protease 13 (USP13) is a class of deubiquitinating enzymes (DUBs) and plays an important role in cellular functions such as cell cycle regulation, DNA damage repair and is implicated in different cell signaling pathways. Studies regarding the role of USP13 in cancer development and progression are divergent and there are no previous data regarding the role of USP13 gene in PTCs. In this study, we investigated the genetic cause of PTC diagnosed in multiple members of a Brazilian family.

Methods: Whole exome sequencing identified a heterozygous missense variant c.1483G>A (p.V495M) in the USP13 gene that fully segregates with the disease. We investigated whether the USP13 c.1483G $>A$ variant found in our PTC patients impacts USP13 protein stability and function in thyroid cancer cells, MDA-T32, by clycloheximide chase assay.

Results: The specific variant (c.1483G>A) preserves protein stability for longer hours compared to the non-mutated USP13 protein. Moreover, USP13 overexpression increased the potential of a single cell to form colony and the USP13 c.1483G>A variant enhanced the effects seen in USP13 overexpression.

Conclusion: Our study suggests that USP13 may enhance tumorigenesis in PTC; in addition, the p.V495M (c.1483G>A) variant may contribute to PTC formation.

\section{Introduction}

Papillary thyroid carcinoma (PTC) is the most common type of endocrine malignancy, representing approximately $85 \%$ of all differentiated thyroid carcinomas $(1,2)$. The incidence of thyroid carcinomas has greatly increased in the last 30 years with a prevalence in women about four times higher than in men (3). This increase is due to high-resolution imaging techniques improvement that led to early detection. However, studies also suggest that external elements as lifestyle changes, environmental conditions, radiation exposure and family history have implications in the increase of thyroid cancer incidence, particularly PTCs $(1,4)$. Despite the high incidence, mortality rates have decreased over the years due to improved management and treatment of the disease (5). Yet, high resistance to standard chemotherapy, extensive resection, recurrence and metastasis can lead to poor outcome (6). In this context, there is still lack of information regarding genetic alterations that lead to PTC onset and progression (7).

In the advent of genetic sequencing techniques, new possibilities for diagnosis of different types of tumors with hereditary origin have emerged. Whole exome sequencing showed to be a potent tool to identify existing variants and became more accessible to clinicians to investigate different diseases (6) and therefore, improved early diagnosis and more prognosis. Particular driver pathogenic variants were found to be associated with different histological subtypes of thyroid cancers. Regarding PTCs, classification by gene expression profile includes BRAF-like pathogenic variants: BRAF ${ }^{V 600 E}$, RET/ PTC 
fusion, NTRK fusions or RAS -like molecular subtypes: RAS, BRAF ${ }^{K 601 E}$, PAX8/PPARG, PTEN, EIF1AX; with $\mathrm{BRAF}^{\mathrm{V} 600 \mathrm{E}}$ being the most common pathogenic variant found in PTCs (40-80\%) (8). Although less common, other genes have showed to have an important role in PTC onset such as DICER1, APC, KTM2D and WDR77(9-11).

Ubiquitin-specific proteases (USPs) are the biggest class of deubiquitinating enzymes (DUBs) and play an important role in cellular functions such as cell cycle regulation, DNA damage repair and different cell signaling pathways. Therefore, due to interactions with different cellular targets, USPs have been associated with tumor development and progression (12). USP13 belongs to the USPs and its function in cancer is controversial. A recent study showed that USP13 may act as an oncogene in cervical cancer (13). In the same direction, it has been shown a pro-tumor role of USP13 in glioblastoma (14). On the other hand, in some studies USP13 gene seems to act as a tumor suppressor by interacting with PTEN signaling in human bladder cancer and oral squamous cell carcinoma (15-17). To our knowledge, there are no prior studies investigating the role of USP13 gene in thyroid cancers in the literature.

In the present work, we investigated the genetic cause of thyroid dysfunctions, including diagnosis of PTCs, Hashimoto thyroiditis and goiter, presented by several individuals from the same family. Whole exome sequencing identified a heterozygous missense variant c.1483G >A (p.V495M) in the USP13 gene that fully segregates with the disease. Since USP13 function in thyroid development and progression is unexplored and little is known about this specific variant in tumor development and progression, we evaluated whether USP13 plays a role in MDA-T32 thyroid cancer cells and whether the variant affects USP13 functionality and stability in these cells.

\section{Methods}

\section{Patients}

Thirteen members of a large Brazilian family were studied. This family was previously screened for genes associated to cancer, including SDHA, SDHB, SDHC, SDHD, BRAF, JUNB, CDKN1B and PTEN (18). In this study, only members without thyroid problems and older than 40 years old were treated as unaffected. All patients were recruited under protocols approved by the Institutional Review Board of the participating institutions and written informed consent was obtained from all participants.

\section{Clinical and DNA studies}

The sampling of epithelial buccal cells was performed as previously described (19). After the collection, $10 \mu$ l of proteinase $K(20 \mathrm{mg} / \mathrm{mL})$ were added to the solution, being left overnight at $65^{\circ} \mathrm{C}$, DNA was purified by adding ammonium acetate $10 \mathrm{M}$, precipitated with isopropanol and resuspended with $50 \mu \mathrm{l}$ of Tris 10mM (pH 7.6) and EDTA 1 mM. Exome sequencing was conducted as we have described elsewhere $(20,21)$. In brief, exome DNA was captured using a SureSelect Human all exome v6 (Agilent Technologies, USA). The 150-bp library was sequenced paired end on the NovaSeq6000 platform (Illumina), and read alignment was done against the human chromosome reference assembly build 37 
(GRCh37, NCBI). Filtering and analysis of the variant calling files was performed using the VarSeq v2.2.2 (Golden Helix Inc., Bozeman, MT) using the hereditary gene panel with modifications adjusted to our studied pedigree. The identified USP13 genetic variant was confirmed through Sanger sequencing in all members of the family carrying it based on the exome sequencing findings.

\section{In silico analyses}

Genetic variants were evaluated by MutationTaster (http://www.mutationtaster.org/), Polymorphism Phenotyping v2 algorithm tool (PolyPhen-2) (http://genetics.bwh.harvard.edu/pph2), SIFT (Sorting Tolerant From Intolerant) algorithm (http://sift.jcvi.org), Mutation Assessor (http://mutationassessor.org/r3), FATHMM (Functional Analysis through Hidden Markov Models v2.3) (http://fathmm.biocompute.org.uk/) and FATHMM MKL (http://fathmm.biocompute.org.uk/fathmmMKL.htm) to predict the possible impact of the amino acid substitution on the structure and function of the corresponding proteins. Variants that were predicted as damaging by more than four in silico tools and also have a frequency in gnomAD less than $1 \%$ were selected for further studies.

\section{Ab initio Modeling}

I-Tasser was used for the ab initio modeling of the native (wild type) and the mutated USP13 deduced amino acid sequences of p.V495M (http://zhanglab.ccmb.med.umich.edu/I-TASSER) (9-11). Predicted models were evaluated for discrete optimized protein energy and root mean square distance (RMSD) score (for the measurement of the average distance between the backbones of the superimposed proteins), and the TM score (to assess topological similarity) was calculated using TM-Align (22-24). The chosen 3-dimensional structures of the native and the mutated structures were further analyzed using PyMOL molecular graphics system (DeLano Scientific; http://pymol.sourceforge.net/).

\section{In vitro Functional Characterization}

\section{Cell culture}

MDA-T32 thyroid cancer cells were acquired from ATCC (CLR-3351) grown in RPMI-1640 medium containing GlutaMAX ${ }^{\mathrm{TM}}$ (72400146, Thermo Fisher, MA, USA) supplemented with $10 \%$ fetal bovine serum (100-106, Gemini Bio Products, CA, USA), and 1\% antibiotic (Penicillin-Streptomycin - 15140-148, Gibco, TX, USA). Cells were incubated in a humidified atmosphere at $37^{\circ} \mathrm{C}$ with $5 \% \mathrm{CO} 2$.

\section{USP13 siRNA}

USP13 siRNA was performed in MDA-T32 cells following manufacturer's protocol. We used Human USP13 on-target plus smart pool siRNA (L-006064-00-0010, Dharmacon, CO, USA) and non-targeting ontarget plus control pool (D-001810-10-05, Dharmacon, CO, USA). Briefly, 3×104 MDA-T32 cells were plated in 12 well plates. The next day, cells were transfected with $1 \mu \mathrm{M}$ of siRNA or control and incubated for $24 \mathrm{~h}$. Medium was replaced by fresh medium for more 24 hours and cells were collected for protein extraction. 


\section{Site directed mutagenesis}

The human USP13 wild type (NM_003940) coding sequence was cloned into the pCMV6-Entry vector with C-terminal Myc-DDK tag (RC202190, Origene, Rockville, MD, USA). The p.V495M variant was introduced into the human USP13 wild type template using the QuikChange Lightning Site-directed Mutagenesis Kit (210518-5, Agilent Technologies, Santa Clara, CA, USA), following the manufacturer's protocol. Insertion of the variant was confirmed by Sanger sequencing. The following mutagenic primers were used:

\section{USP13-Val495Met MUT_F: GCATCAGGTAATCCATCCTCTCCGTGTAGCG}

USP13-Val495Met MUT_R: CGCTACACGGAGAGGATGGATTACCTGATGC

\section{Cycloheximide chase assay}

MDA-T32 cells were seeded into 12-well plates at a density of $3 \times 10^{4}$ cells per well. After $24 \mathrm{~h}$ of incubation, cells were transfected with Effectene Transfection Reagent (301425, Qiagen, Germany) following manufacturer's protocol. $0.5 \mu \mathrm{g}$ of each vector (USP13 wild type and USP $13 \mathrm{p} . \mathrm{V} 495 \mathrm{M}$ variant was used for the transfection. The empty pCMV6-Entry vector was used as a negative control. After $24 \mathrm{~h}$ of transfection, medium was removed, cells were treated with $25 \mu \mathrm{M}$ of cycloheximide (MilliporeSigma, 01810) and incubated for 3, 8 and 24 hours. After each incubation time, cells were washed with PBS and protein was extracted. Experiment was performed in triplicate.

\section{Analysis of protein expression}

Cells seeded in 12 wells plate were resuspended in $35 \mu$ l of ice-cold lysis buffer (Tris- $\mathrm{HCl} 10 \mathrm{mM}, \mathrm{pH} 7,5$, $\mathrm{NaCl} 150 \mathrm{mM}$, EDTA $1 \mathrm{mM}$, EGTA $1 \mathrm{mM}$, SDS $0.1 \%$, Nonidet P-40 1\%) containing a cocktail of protease and phosphatase inhibitors (Sigma-Aldrich PPC1010). The collected cells were incubated for 30 minutes on ice and then centrifuged for 15 minutes at $4^{\circ} \mathrm{C}, 13000 \mathrm{rpm}$. Total protein concentration of supernatant was determined by Pierce ${ }^{\mathrm{TM}}$ BCA Protein Assay (23227, Thermo Scientific, MA, USA), following the manufacturer's protocol. After quantification of protein extracts, $20 \mu \mathrm{g}$ of total proteins of each sample were separated by electrophoresis in $10 \%$ polyacrylamide gel under denaturing conditions (SDS-PAGE). Proteins were then transferred to a nitrocellulose membrane (BioRad) and Western Blot was performed using antibodies against PTEN diluted 1:1000 (SC-25778, Santa Cruz Biotechnology, TX, USA), c-DDK diluted 1:1000 (TA50011-100, Origene, MD, USA), GAPDH diluted 1:2000 (SC-25778, Santa Cruz Biotechnology, TX, USA) and GAPDH diluted 1:2000 (SC-47724, Santa Cruz Biotechnology, TX, USA). Fluorescent secondary antibodies (827-08364 IRDye 800CW Goat anti-Mouse and 926-68073 IRDye 680RD Donkey anti Rabbit, LiCor, USA) diluted 1:20000 and Odyssey CLx Imaging System (Licor, USA) were used to acquire the signal of the bands. Densitometric quantification was performed using ImageJ software and a ratio between protein of interest and GAPDH was calculated and ploted on GraphPad.

\section{Clonogenic survival assay}

$1 \times 10^{3}$ MDA-T32 cells/well were seeded in six well plates for $24 \mathrm{~h}$ and transfected with empty vector (p.CMV6), USP13 wild type and USP13 p.V495M, as described above, for 48h. After incubation time, the 
cell culture medium was removed, and fresh medium was added. Cells were then incubated for 7 days and medium was changed every two days. In the end point of the experiment, medium was removed, and colonies were fixed with a solution containing $0.05 \% \mathrm{w} / \mathrm{v}$ of crystal violet, $1 \%$ of formaldehyde and $1 \%$ methanol in PBS. Fixed colonies were photographed at a magnification of $4 x$. One milliliter of acetic acid $10 \%$ was added to each well and plates were shaken for 15 minutes to discolor the plaque. $200 \mathrm{~mL}$ of the violet stain solution removed from the plate was transferred to a 96-well plate and read on a spectrophotometer at an absorbance of $590 \mathrm{~nm}$. The intensity of staining is proportional to the number of colonies established after treatment. Experiment was performed in triplicate.

\section{Cell viability assay}

MDA-T32 cells were seeded into $96-$ well plates at a density of $4 \times 10^{3}$ cells per well and incubated for $24 \mathrm{~h}$. Cells were then transfected with the non-target control or siRNA as described above and incubated for 0 , 24, 48 and 72 hours. At each time point, MTT (3-(4,5-dimethylthiazol-2-yl)-2,5-diphenyltetrazolium salt) was added to a final concentration of $0.5 \mathrm{mg} / \mathrm{mL}$ into the culture media. After $3 \mathrm{~h}$ of incubation with MTT, medium was removed and $200 \mathrm{~mL}$ of isopropanol containing acetic acid $0.04 \mathrm{M}$ was added into the wells and plate was gently shaken to solubilize the product reduced by MTT. Absorbance was measured at 570nm.

\section{Statistical analysis}

Statistical analysis was performed using Student's t-test or One-way ANOVA with multiple comparisons tool. Data are expressed as mean \pm SEM. Differences between mean values were considered significant when $p<0.05$.

\section{Results}

\section{Genetic Analysis}

Filtering of the variant calling files, performed in the VarSeq v2.2.2 (Golden Helix Inc., MT, USA) using the hereditary gene panel, revealed a heterozygous missense variant in the USP13 gene c.1483G >A (p.V495M). Initially, we included into the genetic analysis the following patients (Affected: I-1, I-2, II-3, II-4, II-3, II-9; Unaffected: I-3, II-2, II-8, III-2) (Fig. 1). This selection was based on the stringiest inclusion criteria based on the phenotype. The results of this analysis picked the USP13 genetic variant c.1483G > A (p.V495M) out of 83 genetic variants as the only one that segregates with the disease in this family. The second step in the genetic analysis included the addition of more family members with thyroid related issues (Goiter, Hashimoto Thyroiditis, etc.) (Affected: I-1, I-2, II-3, II-4, II-3, II-5, II-7, II-9, III-1; Unaffected: I-3, II-2, II-8, III-2) (Fig. 1). This broader genetic analysis revealed 78 genetic variants present in affected members. The variant c.1483G > A (p.V495M) in the USP13 gene showed to segregate not only in family members affected with PTC, but also in members affected with other thyroid problems.

\section{Predicted damaging variant in USP13 was found segregating with the disease}


A heterozygous missense variant was detected in USP13 gene c.1483G > A (p.V495M) that fully segregates with the disease. The genealogic tree of the family shows that individuals with the USP13 variant either developed PTC, or Hashimoto thyroiditis or goiter (Fig. 1b). This variant has an allele frequency of 0.000302 (gnomAD Exomes v2.1.1) and it is predicted to be damaging in four out of six in silico analysis tools used (Polyphen-2, MutationTaster, MutationAssessor and FATHMM MKL). This missense variant is located to the USP domain of the USP13 protein and the mutated amino acid is evolutionarily highly conserved among species (Fig. 2a).

\section{In silico modeling suggests that USP13 c.1483G > A (p.V495M) variant may cause protein structural perturbations}

The TM score of the alignment between that native (wild type) and the mutant protein (p.V495M) was 0.93687 and the RMSD value was 2.98 , indicating potential pathogenicity for the identified missense variant. RMSD values greater than 0.15 are considered as an indicator for significant structural perturbations, which could have functional implications for the protein function (22). The 3-dimensional effect of the p.V495M variant that is located at the USP domain was defined using ab initio modeling. As shown in Fig. 2B, the alignment of the ab initio native and mutant model reveals that the variant at the position 495 may change the folding of the protein as the new amino acid Met contains a sulfur that is predicted to interact with the aromatic residue tyrosine in position 497 as indicated by the dotted line. Moreover, this variant is located in the USP domain, a critical domain for DUBs, and therefore may have an impact in the protein function.

\section{USP13 does not affect PTEN stabilization in MDA-T32 thyroid cancer cells}

Studies in different types of tumors already demonstrated that USP13 stabilizes PTEN, the second most frequent mutated gene in human cancers $(15-17,25,26)$. Since there are no records in the literature regarding signaling of USP13 in PTCs, we checked whether USP13 would play a role in PTEN stabilization. We evaluated PTEN expression in USP13 siRNA MDA-T32 cell lines. Our results show that the absence of USP13 does not affect expression of PTEN in this cell lineage (Fig. 3a-c).

\section{Silencing USP13 in MDA-T32 cells decreases cell proliferation and colony formation}

The ability of cells to constantly proliferate without an external stimulus is one of the main aspects of cancer cells. The capacity of a single cell to migrate and stablish colonies in a distant site of its previous microenvironment is also another important feature acquired by cancer cells (27). We investigated whether USP13 would have a function on proliferation and clonogenic capacity of thyroid cancer cells. USP13 siRNA was performed in MDA-T32 cells and our results show that reduced expression of USP13 decreases the capacity of cells to form colonies (Fig. 3d-e) and leads to decreased cell proliferation rates after 72 hours of USP13 siRNA transfection (Fig. 3f) 


\section{USP13 c.1483G > A variant sustains protein stability for longer periods}

The cycloheximide chase assay enables to observe degradation kinetics of proteins and therefore is a good tool to study protein stability (28). By treating wild type and USP13 c.1483G > A mutated cells with cycloheximide, an inhibitor of protein synthesis, we were able to evaluate the dynamics of USP13 degradation when MDA-T32 thyroid cells present the variant. After 8 hours of cycloheximide treatment, expression of USP13 in wild type cells is significantly decreased, while in cells transfected with the USP13 c. $1483 \mathrm{G}>\mathrm{A}$ variant, this effect is seen after 24 hours of cycloheximide treatment (Fig. 4b-c). These results suggest that the presence of the variant have a significant impact on the USP13 stability and consequently on its targets, as USP13 will be active in the cells for longer periods.

\section{USP13 c.1483G>A variant increases MDA-T32 thyroid cancer cells colony formation}

As studies available in the literature regarding the role of USP13 in cancer development and progression are controversial, with evidence of USP13 acting as an oncogene or as a tumor suppressor, depending on the type of cells, it is hard to predict whether this variant would be, in fact, contributing to the survival of thyroid cancer cells. To answer this question, we evaluated the effect of the variant in the capacity of colony formation of MDA-T32 cells. Our results show that the overexpression of USP13 gene increased the capacity of the cells to form colonies. Interestingly, the presence of the variant, USP13 c.1483G >A (p.V495M), drastically increased this effect (Fig. 4d-e), suggesting that, in thyroid cancer cells, USP13 might act as an oncogene and, in the presence of the variant, the clonogenic capacity of the cells are enhanced.

\section{Discussion}

Attempting to investigate the genetic cause of PTC in multiple members of the same family, we found an association of the USP13 c.1483G > A; p.V495M variant with PTC development. Individuals that carry the variant either developed PTC or other thyroid dysfunctions such as Hashimoto thyroiditis or goiter, suggesting that the onset of these thyroid dysfunctions could be related to genetic factors (29). It is important to mention that carriers of the variant that did not developed PTC may develop it later since the age of onset may not have been reached yet.

Ubiquitination is an essential post-translational modification (PTM) event that regulates protein function, localization and turnover, contributing to sustain cellular homeostasis. The ubiquitination process can be reversed by ubiquitin-specific proteases (USPs). Thus, USPs play an important role in cell signaling, control of protein degradation and tumorigenesis $(12,30)$. USP13 is a subtype of USP and its role in tumor development and progression is controversial. Here, we report a variant in the USP13 gene (c.1483G > A; p.V495M) that is present in multiple members of the same family diagnosed with PTC. 
Studies attributed a tumor suppressor role to USP13 gene by showing that USP13 mediates PTEN signaling in different types of cancers (15-17). In our study, silencing USP13 gene in MDA-T32 thyroid cancer cell lines did not interfere on PTEN expression (Fig. 3a-c). Therefore, other pathways could be involved in USP13 signaling of MDA-T32 cells. On the other hand, decreased expression of USP13 in MDA-T32 thyroid cancer cells led to a less severe phenotype, such as decreased proliferation rates and clonogenic capacity, suggesting that USP13 may play a pro-tumor role in PTCs (Fig. 3d-f).

Due to the segregation of the USP 13 c.1483G > A; p.V495M variant within individuals that presented thyroid disfunctions reported in this study, and the putative pro-tumor role of USP13 seen in vitro, we hypothesized that it could be a gain-of-function variant. In fact, we found that the presence of the variant increased USP13 half-life compared to the wild type protein in MDA-T32 cells (Fig. 4b-c). Based on available data in the literature, the role of USP13 in tumorigenesis is ambiguous. A few studies suggest a tumor suppressor role for USP13: USP13 showed to stabilize PTEN, a potent tumor suppressor, in breast, bladder cancers, and oral squamous cell carcinomas (15-17). On an opposite direction, other studies show that USP13 may act as an oncogene. For instance, USP13 stabilizes c-Myc that leads to an increased proliferation of glioma cells (14) and Mcl-1, which seems to increase proliferation of lung, ovarian and cervical cancer cells $(13,31)$. Moreover, silencing USP13 decreased cell proliferation in lung metastasis of hepatocellular carcinoma (32) and its overexpression is correlated with poor prognosis and chemoresistance of ovarian cancer (33). USP13 was also showed to interact with aurora kinase B and disrupt cell cycle regulation (34).

Our in vitro analysis showed that the USP13 c.1483G > A; p.V495M variant preserves protein stability in MDA-32 thyroid cancer cells compared to non-mutated USP13. Supporting the pro-tumor role of USP13 previously described in other types of cancers, we demonstrated that USP13 overexpression increases the capability of a single cell to form colonies and that the c.1483G > A; p.V495M variant enhances the effects seen in the USP13 overexpression, causing an even more severe phenotype compared to the wild type protein (Fig. 4d-e). To our knowledge, there is no data in the literature regarding the correlation of USP13 and PTCs. Herein, our genetic analysis and in vitro studies provided evidence that the presence of the USP13 c.1483G > A; p.V495M variant contributes to PTC onset and that USP13 may act as an oncogene in PTCs. Taken together, we show two different scenarios in this study: 1. Patients that present the heterozygous USP13 c.1483G > A variant developed thyroid dysfunctions such as PTC, Hashimoto thyroidits or goiter. 2. In vitro assays present evidences that the homozygous USP13 c.1483G > A can favor tumor growth. By performing the in vitro assay, we were able to better understand the role of USP13 in PTCs while seeing heterozygous patients developing the disease opens a discussion on the importance of genetic screening for early diagnosis of PTCs.

A limitation of the study is the availability of tissues to analyse the prevalence of the variant in individuals with PTCs, as well as availability of tissues to evaluate and compare USP13 expression in neoplastic tissues with contralateral non neoplastic samples. However, because the incidence of PTCs is constantly increasing (3), this study may have a great impact on early diagnosis and prognosis of the disease, opening new avenues for follow up of individuals that carry USP13 genetic variants in regard to 
thyroid dysfunction. Furthermore, this study brings the first evidence that USP13 might be an oncogene in the context of PTCs and may also cause thyroid dysfunctions such as Hashimoto thyroidits and goiter.

\section{Declarations}

Funding: This work was funded by the NIH Intramural Grant Z01-HD008920-01 of the Eunice Kennedy Shriver National Institute for Child Health \& Human Development (NICHD), Division of Intramural Research (DIR) to Dr. Constantine A. Stratakis.

Disclosure summary: Dr. Stratakis holds patents on the function of the PRKAR1A, PDE11A, and GPR101 genes and related issues; his laboratory has also received research funding on GPR101 and its involvement in acromegaly and/or gigantism, abnormal growth hormone secretion and its treatment by Pfizer, Inc.; Dr. Faucz holds patent on the GPR101 gene and/or its function; The other authors have nothing to disclose.

\section{References}

1. Rangel-Pozzo A, Sisdelli L, Cordioli MIV, Vaisman F, Caria P, Mai S, et al. Genetic Landscape of Papillary Thyroid Carcinoma and Nuclear Architecture: An Overview Comparing Pediatric and Adult Populations. Cancers (Basel). 2020;12(11).

2. Macerola E, Poma AM, Vignali P, Basolo A, Ugolini C, Torregrossa L, et al. Molecular Genetics of Follicular-Derived Thyroid Cancer. Cancers (Basel). 2021;13(5).

3. Krajewska J, Kukulska A, Oczko-Wojciechowska M, Kotecka-Blicharz A, Drosik-Rutowicz K, Haras-Gil $\mathrm{M}$, et al. Early Diagnosis of Low-Risk Papillary Thyroid Cancer Results Rather in Overtreatment Than a Better Survival. Front Endocrinol (Lausanne). 2020;11:571421.

4. Abdullah MI, Junit SM, Ng KL, Jayapalan JJ, Karikalan B, Hashim OH. Papillary Thyroid Cancer: Genetic Alterations and Molecular Biomarker Investigations. Int J Med Sci. 2019;16(3):450-60.

5. Raposo L, Morais S, Oliveira MJ, Marques AP, Jose Bento M, Lunet N. Trends in thyroid cancer incidence and mortality in Portugal. Eur J Cancer Prev. 2017;26(2):135-43.

6. Fang Y, Ma X, Zeng J, Jin Y, Hu Y, Wang J, et al. The Profile of Genetic Mutations in Papillary Thyroid Cancer Detected by Whole Exome Sequencing. Cell Physiol Biochem. 2018;50(1):169-78.

7. Pozdeyev N, Gay LM, Sokol ES, Hartmaier R, Deaver KE, Davis S, et al. Genetic Analysis of 779 Advanced Differentiated and Anaplastic Thyroid Cancers. Clin Cancer Res. 2018;24(13):3059-68.

8. Nylen C, Mechera R, Marechal-Ross I, Tsang V, Chou A, Gill AJ, et al. Molecular Markers Guiding Thyroid Cancer Management. Cancers (Basel). 2020;12(8).

9. Ghossein CA, Dogan S, Farhat N, Landa I, Xu B. Expanding the spectrum of thyroid carcinoma with somatic DICER1 mutation: a survey of 829 thyroid carcinomas using MSK-IMPACT next-generation sequencing platform. Virchows Arch. 2021. 
10. Nieminen TT, Walker CJ, Olkinuora A, Genutis LK, O'Malley M, Wakely PE, et al. Thyroid Carcinomas That Occur in Familial Adenomatous Polyposis Patients Recurrently Harbor Somatic Variants in APC, BRAF, and KTM2D. Thyroid. 2020;30(3):380-8.

11. Zhao Y, Yu T, Sun J, Wang F, Cheng C, He S, et al. Germ-line mutations in WDR77 predispose to familial papillary thyroid cancer. Proc Natl Acad Sci U S A. 2021;118(31).

12. Cruz L, Soares P, Correia M. Ubiquitin-Specific Proteases: Players in Cancer Cellular Processes. Pharmaceuticals (Basel). 2021;14(9).

13. Morgan EL, Patterson MR, Barba-Moreno D, Scarth JA, Wilson A, Macdonald A. The deubiquitinase (DUB) USP13 promotes Mcl-1 stabilisation in cervical cancer. Oncogene. 2021;40(11):2112-29.

14. Fang X, Zhou W, Wu Q, Huang Z, Shi Y, Yang K, et al. Deubiquitinase USP13 maintains glioblastoma stem cells by antagonizing FBXL14-mediated Myc ubiquitination. J Exp Med. 2017;214(1):245-67.

15. Man X, Piao C, Lin X, Kong C, Cui X, Jiang Y. USP13 functions as a tumor suppressor by blocking the NF-kB-mediated PTEN downregulation in human bladder cancer. J Exp Clin Cancer Res. 2019;38(1):259.

16. Qu Z, Zhang R, Su M, Liu W. USP13 serves as a tumor suppressor via the PTEN/AKT pathway in oral squamous cell carcinoma. Cancer Manag Res. 2019;11:9175-83.

17. Zhang J, Zhang P, Wei Y, Piao HL, Wang W, Maddika S, et al. Deubiquitylation and stabilization of PTEN by USP13. Nat Cell Biol. 2013;15(12):1486-94.

18. Accordi ED, Xekouki P, Azevedo B, de Alexandre RB, Frasson C, Gantzel SM, et al. Familiar Papillary Thyroid Carcinoma in a Large Brazilian Family Is Not Associated with Succinate Dehydrogenase Defects. Eur Thyroid J. 2016;5(2):94-9.

19. Azevedo LF, Pecharki GD, Brancher JA, Cordeiro CA, Jr., Medeiros KG, Antunes AA, et al. Analysis of the association between lactotransferrin (LTF) gene polymorphism and dental caries. J Appl Oral Sci. 2010;18(2):166-70.

20. Drougat L, Settas N, Ronchi CL, Bathon K, Calebiro D, Maria AG, et al. Genomic and sequence variants of protein kinase $A$ regulatory subunit type 1 beta (PRKAR1B) in patients with adrenocortical disease and Cushing syndrome. Genet Med. 2021;23(1):174-82.

21. Espiard S, Knape MJ, Bathon K, Assie G, Rizk-Rabin M, Faillot S, et al. Activating PRKACB somatic mutation in cortisol-producing adenomas. JCI Insight. 2018;3(8).

22. Mistri M, Tamhankar PM, Sheth F, Sanghavi D, Kondurkar P, Patil S, et al. Identification of novel mutations in HEXA gene in children affected with Tay Sachs disease from India. PLoS One. 2012;7(6):e39122.

23. Roy A, Yang J, Zhang Y. COFACTOR: an accurate comparative algorithm for structure-based protein function annotation. Nucleic Acids Res. 2012;40(Web Server issue):W471-7.

24. Zhang Y. I-TASSER server for protein 3D structure prediction. BMC Bioinformatics. 2008;9:40.

25. Xiang S, Fang J, Wang S, Deng B, Zhu L. MicroRNA135b regulates the stability of PTEN and promotes glycolysis by targeting USP13 in human colorectal cancers. Oncol Rep. 2015;33(3):1342-8. 
26. Yin Y, Shen WH. PTEN: a new guardian of the genome. Oncogene. 2008;27(41):5443-53.

27. Hanahan D, Weinberg RA. Hallmarks of cancer: the next generation. Cell. 2011;144(5):646-74.

28. Buchanan BW, Lloyd ME, Engle SM, Rubenstein EM. Cycloheximide Chase Analysis of Protein Degradation in Saccharomyces cerevisiae. J Vis Exp. 2016(110).

29. Lee YJ, Cho YJ, Heo YJ, Chung EJ, Choi YH, Kim JI, et al. Thyroid nodules in childhood-onset Hashimoto's thyroiditis: Frequency, risk factors, follow-up course and genetic alterations of thyroid cancer. Clin Endocrinol (Oxf). 2021;95(4):638-48.

30. Young MJ, Hsu KC, Lin TE, Chang WC, Hung JJ. The role of ubiquitin-specific peptidases in cancer progression. J Biomed Sci. 2019;26(1):42.

31. Zhang S, Zhang M, Jing Y, Yin X, Ma P, Zhang Z, et al. Deubiquitinase USP13 dictates MCL1 stability and sensitivity to BH3 mimetic inhibitors. Nat Commun. 2018;9(1):215.

32. Gao S, Chen T, Li L, Liu X, Liu Y, Zhao J, et al. Hypoxia-Inducible Ubiquitin Specific Peptidase 13 Contributes to Tumor Growth and Metastasis via Enhancing the Toll-Like Receptor 4/Myeloid Differentiation Primary Response Gene 88/Nuclear Factor-kappaB Pathway in Hepatocellular Carcinoma. Front Cell Dev Biol. 2020;8:587389.

33. Kim W, Zhao F, Gao H, Qin S, Hou J, Deng M, et al. USP13 regulates the replication stress response by deubiquitinating TopBP1. DNA Repair (Amst). 2021;100:103063.

34. Esposito M, Akman HB, Giron P, Ceregido MA, Schepers R, Ramos Paez LC, et al. USP13 controls the stability of Aurora B impacting progression through the cell cycle. Oncogene. 2020;39(37):6009-23.

\section{Figures}

\section{Figure 1}

USP13 variant analysis. (a) Genealogical tree and USP13 c.1483G>A; p.V495M variant analysis. Open symbols represent individual that are wild type for USP13. Filled symbols indicate individuals that are carriers of the USP13 c.1483G >A variant. Black filled symbols indicate individuals diagnosed with papillary thyroid carcinoma, gray symbols indicate individuals diagnosed with other thyroid problems (Hashimoto Thyroiditis or goiter). Truncated symbols indicate deceased individuals. (b) Electropherogram of part of USP13 sequence including the position c.1483 of each member represented in the genealogical tree. Diagnosis of papillary thyroid carcinoma or other thyroid complications are indicated above the electropherogram.

\section{Figure 2}


In silico prediction of the USP13 structure. (a) Protein sequence alignment evidencing the USP13 p.V495M variant within the USP13 domain and amino acid residue conservation among different species. (b) I-TASSER predicted ab-initio model of USP13 c.1483G>A; p.V495M variant. Secondary structure of USP domain with a focus on the USP13 c.1483G>A; p.V495M variant.

\section{Figure 3}

USP13 siRNA in MDA-T32 cells. (a) USP13 and PTEN expression analysis of MDA-T32 cells transfected with USP13 siRNA performed by western blot. (b) Band densitometry quantification of USP13 and (c) PTEN normalized by the non-target siRNA. ${ }^{*} p>0.0001$. (d) Representative images of clonogenic assay to access the capacity of MDA-T32 cells transfected with USP13 siRNA for 48 hours to establish colonies. Cells were stained with Crystal Violet after 7 days of transfection. (e) Absorbance plot of the colony staining. * $p>0.0001$. (f) Cell viability accessed by MTT assay in MDA-T32 cells transfected with USP13 siRNA for 24,48 and $72 \mathrm{~h} .{ }^{*} \mathrm{p}=0.013$.

\section{Figure 4}

USP13 c.1483G>A; p.V495M functional assessment. (a) Electropherogram of part of USP13 sequence including the position c.1483 in the pCMV6 USP13 WT and mutant vector. (b) Cycloheximide chase assay test performed in MDA-T32 cells transfected with pCMV6 USP13 WT and mutant vector. After 24 hours of transfection, cells were treated with cycloheximide for additional 3, 8 and 24 hours. Representative blots of USP13 expression analyzed by western blot. (c) Band densitometry quantification of USP13 expression for all the different times comparing USP13 WT and mutant. * $\mathrm{p}=0.01$ (d) Representative images of clonogenic assay to access the capacity of MDA-T32 cells transfected with USP13 WT or USP13c.1483G >A for 48 hours to establish colonies. Cells were stained with Crystal Violet after 7 days of transfection. (e) Absorbance plot of the colony staining. ${ }^{*} p>0.001$. WT: wild type. 